\title{
Construction of Water Environment Carrying Capacity Evaluation Model in Erhai River Basin
}

\author{
Liu Wei-Hong ${ }^{1,2}$, Zhang Cheng-Gui ${ }^{1,3}$, Gao Peng-Fei ${ }^{1,3}$,Liu Heng ${ }^{1,3}$, Song \\ Yan-Qiu ${ }^{1}$, Huang Bi-Sheng ${ }^{4}$, Yang Jian-Fang ${ }^{* 1,5}$
}

${ }^{1}$ The Key Laboratory of Medical Insects and Spiders Resources for Development \& Utilization at Yunnan Province; Dali University, Dali 671000, Yunnan Province, China;

${ }^{2}$ The Libraries of Dali University, Dali 671000, Yunnan Province, China;

${ }^{3}$ National-local Joint Engineering Research Center of Entomoceutics, Dali 671000, Yunnan Province, China

${ }^{4}$ Department of Agriculture and Biological Sciences, Dali University, Dali 671003, People's Republic of China

${ }^{5}$ School of Foreign Languages, Dali University, Dali 671000, Yunnan Province, China;

Liu Wei-Hong and Zhang Cheng-Gui have contributed equally to this work.

${ }^{*}$ Corresponding author:

Associate Professor Yang Jian-Fang

The Key Laboratory of Medical Insects and Spiders Resources for Development \& Utilization at Yunnan Province; Dali University, Dali 671000, Yunnan Province, China;

E-mail: liuwhong@dali.edu.cn

Keywords: Water environment carrying capacity, Evaluation model, Erhai River basin.

\begin{abstract}
With the acceleration of urbanization and increasing energy consumption in China, the intensity of pollution emission the pollution load is increasing significantly. In many rivers, the main pollutant load is much more than the environmental capacity of the water, resulting in the destruction of the structure and function of the river basin. This paper puts forward the concept of water environment carrying capacity, and constructs the basic model of calculating water environment carrying capacity, and then takes ErhaiRiver as an example to calculate the carrying capacity of water environment in order to provide reference for relevant researchers.
\end{abstract}

\section{Concept of Water Environment Carrying Capacity}

With economic and social development, parts of the ecosystem in the rivers and lakes have degraded. In order to improve the ecosystem in the rivers and lakes, we proposethe new theory of ecosystem in the rivers and lakes. Formed in the ecosystem in the rivers and lakes the continuity of the process based, longitudinal arranged in the river lake, is of a mosaic of specific ecological functions of plaque composition. Based on the analysis of the basic characteristics of river basin water ecological system, combined with other related disciplines, the related research results of water environmental carrying capacity, water resources carrying capacity, ecological carrying capacity, ecological protection as the goal, defining the basic connotation of river basin water ecological environment, the water ecological carrying capacity is defined as: water ecological carrying capacity is in the water ecological function area, and can support the ecological system structure and function and sustainable development. The connotation of water ecological carrying capacity of the river basin is mainly from the connotation of ecological connotation and economic society.

River Basin water ecological carrying the ecological connotation of force can achieve maximum ecological development of aquatic ecosystem health scale.Lakes and river ecological system in the process of basin of matter and energy cycle has the important function of habitat, filtering, shielding, a channel. However, due to the rapid development of human society and economy, the structure and 
function of the internal ecosystem of the river basin have been destroyed, and the stability of the ecosystem cannot be guaranteed. Therefore, there is an ecological limit regulation in the game of the river basin water ecological system and social economic system. The ecological limit of water ecosystem cannot be separated from the value of the specific area population and the specific utility needs. In the same water resource utilization and sewage discharge, the social economic capacity or scale will be different, which will make the ecological carrying capacity of water has a social and economic connotation. In recent years, the contradiction between ecologicaland economy are becoming more and more obvious, and the understanding of the economic connotation should more reflect the relationship and mechanism between the main body and object of the river basin water ecological carrying capacity. We should adjust the internal structure, growth mode and spatial distribution of the social economic development.

\section{Evaluation Model of Water Environment Carrying Capacity}

This paper constructs basin water environment bearing force calculation method of the system, development of reaction ErhaiRiver water dynamics. Lake water dynamics and water quality model and in the model as a calculation tool, according to the water environment bearing capacity calculation method of system, calculate the Erhai River, the main pollutants of water environment bearing capacity.

Water Quality Coefficient. The equilibrium concentration field can be regarded as the linear superposition of the different pollution sources. We assume $C_{i}$ is the separate concentration fielding of number i pollution sources $Q_{i} . C_{b i}$ is the background concentration of the water quality control point, the concentration field in the presence of $n$ pollution sources:

$$
C(x, y, t)=\sum_{i=1}^{n} C_{i}+C_{b i}
$$

At the same time, the concentration field formed by a strong value can be regarded as a result of linear superposition of several units, which is based on the following relationship:

$$
C i=P_{i} \cdot Q_{i}
$$

In the formula, $P_{i}$ is the concentration fieldwhen $Q_{i}=1$. We define $P_{i}$ as the response factor. It represents the response of water quality to a certain point. Obviously, because of the interaction of various environmental factors, the distribution of the value of the water in the lake area changes with the location of the response coefficient field. Response coefficient is a quantitative relationship between water quality and pollution sources in the lake area, which is based on the principle of mass conservation, which is the basis of the calculation of water environmental carrying capacity.

Pollution Sharing Rate. Pollution Sharing rate refers to the impact of the pollution sources in the water area of the total pollution impact of the share (percentage), that is:

$$
r_{i}=C_{i} / \sum_{i=1}^{n} C_{i}
$$

Sharing rate indicates that the degree of the pollution sources is responsible for water pollution. It is obvious that the sharing rate has the following characteristics: the same source of pollution is different in different regions, and different pollution sources are also different in different regions.

Allowable Discharge Amount. The water quality control standard and response coefficient field and sharing rate can be calculated to meet the water quality control objectives of the conditions to meet the water quality control objectives. It can be carried out in the following steps: Step 1: the response factor field of each point source is calculated by the equation. Step two: calculate the share of each point source field. Step three: the calculation of the amount of each point source.

$$
\begin{gathered}
C_{0 i}=r_{i}\left(C_{0}-C_{b i}\right) \\
Q_{0 i}=\frac{C_{0 i}}{P_{i}}=\frac{r_{i}\left(C_{0}-C_{b i}\right)}{P_{i}}
\end{gathered}
$$


$Q_{0 i}$ isthe allowable emission intensity of the i point source under the condition of water quality objectives. The total allowable discharge amount of water in the lake area is

$$
Q_{0}=\sum_{i=1}^{n} Q_{0 i}
$$

Decreasing Rate. Therefore, the decreasing amount of i point source is

$$
\Delta Q_{i}=Q_{i}-Q_{0 i}
$$

In that formula, $Q_{i}$ is the current emissions; if $\Delta Q_{i}>0$, it means we should decrease the current amount according to the standard decreasing rate. If ${ }^{\Delta Q_{i}}<0$, it means we should not decrease the current amount

Conditions of Evaluation Model. To calculate the number of parameters, it is necessary to use Lake Hydrodynamics and water quality model. The ErhaiRiver water dynamics and water quality model using the depth averaged two-dimensional unsteady model. The main calculation conditions are as follows: the water level of the lake is an important factor to the water environment carrying capacity of the lake, which is the basic condition for the calculation of the water environment carrying capacity of Erhai. According to the Autonomous Prefecture of Erhai management regulations, the minimum operating water level in Erhai is 1964.30 meters, the highest level of 1966 meters. At the same time according to the Erhai 1997 to 2008 the average monthly water level can be obtained in the past 12 years, the average operating level of Erhai is about 1964.69 meters. So in the calculation of Erhai Lake water environment bearing capacity, we should selectoperating for many years the average level 1964.69 as a model for computing the characteristics of water level of basic conditions. The water environmental carrying capacity of the lake has a certain impact on the water level of the lake, and the water environment carrying capacity of Erhai is also considered. According to the foregoing, Erhai Lake of various typical hydrological years into the lake water respectively is: Feng water in 14.39 x $108 \mathrm{~m} 3$, flat water years 11.64 x 108 in dry years $7.632 \times 108 \mathrm{~m} 3$. According to the Erhai wind field characteristics, typical wind parameters calculation of bearing capacity of Erhai water environment is wind speed $4.1 \mathrm{~m} / \mathrm{swith}$ the southwest wind direction.

\section{Empirical Research of Erhai River Basin}

In the ancient literature,ErhaiRiver has called for the West River, located in the suburb of Dali in Yunnan Province. It is the second largest freshwater lake in Yunnan Province.Erhai Lake originates from north Eryuan, $42.58 \mathrm{~km}$ long, and $9.0 \mathrm{~km}$. The maximum width, Lake area of 256.5 square kilometers, lake with an average depth of 10 meters, maximum lake depth of 20 meters. Erhai has two outlets: in near the town of Shimonoseki, the outflow of Xierhe; "lead Er into the bin". Erhai is one of the "Dali four king" romantic themes "Erhai" where. It is said to be named "Erhai" because of the shape of an ear". Erhaihas good water quality, abundant marine resources, but also a Yili scenery scenic area. Erhai, although known as the sea, but in fact is a lake, is said to be because the Bai people have not seen the sea, in order to express the yearning for the sea, so called Erhai. According to the calculation method of water environmental carrying capacity and the calculation condition, the water environmental carrying capacity of the water environment of Erhai in the years of the average operating water level is obtained. As shown in the table 1.

Table 1 Water Environment Carrying Capacity of Erhai River

\begin{tabular}{|c|l|r|r|}
\hline \multirow{2}{*}{$\begin{array}{c}\text { Characteristic } \\
\text { water level(m) }\end{array}$} & \multicolumn{1}{c|}{$\begin{array}{c}\text { Planning } \\
\text { Objective }\end{array}$} & \multicolumn{2}{|c|}{ Primary Pollutants } \\
\cline { 3 - 4 } & & \multicolumn{2}{c|}{ TP } \\
\hline \multirow{2}{*}{1964.69} & I & 2063.8 & 115.5 \\
\cline { 2 - 4 } & II & 4127.5 & 231 \\
\cline { 2 - 4 } & III &
\end{tabular}




\section{Application of Water Environment Carrying Capacity of Erhai}

Based on the above analysis, the main problem in Erhai isthe land use zoning of the river basin. We carry outthe Erhai River Basin and water pollution prevention and control planning objectives and requirements of the Erhai and its drainage basin to carry out land use zoning based on the principle. In the area, the following principles shall be followed: pollution prevention and control division and planning functional zoning, and the principle of the distribution of pollution sources, and the relevant provisions of the Erhai protection ordinance. Comprehensive prevention and control division according to the characteristics of the Erhai river basin topography, pollution sources distribution characteristics, according to the principle of division, the Erhai river basin is divided into four tracts. Fengyi town of urban domestic and industrial development and pollution; failure wave Luo river basin mountain, on both sides of the village and agricultural production also bring certain pollution watershed division industry development of four kinds of function control area. In the west of the East Lake Hai bin Lu, Haixi from Erhai Lake of the highest water level line 100 meters ranging from within, in West Lake the highest water level of 100 meters ranging from within, draw a red line as basin industry prohibition of the development zone. The prohibition of the development zone is the distance from the lake and the river system is the nearest region, ecological sensitive area of lakeside zone. The West of Erhai, the scope of the National Road 214 and the mountain conservation forest are a comprehensive development zone of the river basin.

This work was supported by the Erhai special project of Dali University (No. KYRH201010 ,No. KYRH201014), the National Natural Science Foundation of China (No. 81102806, No. 81260676, No. 81360679), the opening project of the State Key Laboratory of Microbial Resources, Institute of Microbiology,Chinese Academy of Sciences (No.SKLMR-20100602),Yunnan Provincial Natural Science Foundation (2011FZ166).

\section{References}

[1] F. Huang, Research of Agricultural Land Use Zoning Based on the Bearing Capacity of Water Environment Erhai Lake Basin. Central China Normal University, 2012.

[2] S.L. Wang, Research on Total Amount Control of Pollutant Based on Water Ecological Carrying Capacity in Basin. Wuhan University, 2014.

[3] J.Y. Wang, J.H. Du. P.R. Lu, Y. Zhang, J.B. Mu, The Evaluation of Water Eco-Environmental Carrying Capacity in Shandong Province, Journal of Shandong University (Engineering Science), 5(2008)94-98.

[4] C. Zeng, Y.F. Liu. W.S. Zhang, D.W. Tang, Origination and Development of the Concept of Aquatic Ecological Carrying Capacity in the Basin, Resources and Environment in the Yangtze Basin, 2(2011)203-209.

[5] Meng L, Chen Y, Li W, et al. Fuzzy Comprehensive Evaluation Model for Water Resources Carrying Capacity in Tarim River Basin, Xinjiang, China. Chinese Geographical Science, 2009, 19(1):89-95.

[6] Yan Z, Xia J, Zuo Q, et al. Computing System Construction of Water Environment Carrying Capacity in Huaihe River Basin[J]. Resources Science, 2009, 31(7):1150-1157. 